\title{
Multiobjective Tree-structured Parzen Estimator for Computationally Expensive Optimization Problems
}

\author{
Yoshihiko Ozaki \\ AI Research Center, AIST \& GREE, Inc. \\ Tokyo, Japan \\ ozaki-y@aist.go.jp \\ Shuhei Watanabe \\ AI Research Center, AIST \\ Tokyo, Japan \\ shuhei.watanabe@aist.go.jp
}

\author{
Yuki Tanigaki \\ AI Research Center, AIST \\ Tokyo, Japan \\ tanigaki.yuki@aist.go.jp \\ Masaki Onishi \\ AI Research Center, AIST \\ Tokyo, Japan \\ onishi@ni.aist.go.jp
}

\begin{abstract}
Practitioners often encounter computationally expensive multiobjective optimization problems to be solved in a variety of real-world applications. On the purpose of challenging these problems, we propose a new surrogate-based multiobjective optimization algorithm that does not require a large evaluation budget. It is called Multiobjective Tree-structured Parzen Estimator (MOTPE) and is an extension of the tree-structured Parzen estimator widely used to solve expensive single-objective optimization problems. Our empirical evidences reveal that MOTPE can approximate Pareto fronts of many benchmark problems better than existing methods with a limited budget. In this paper, we discuss furthermore the influence of MOTPE configurations to understand its behavior.
\end{abstract}

\section{CCS CONCEPTS}

- Applied computing $\rightarrow$ Multi-criterion optimization and decision-making; • Theory of computation $\rightarrow$ Mathematical optimization; • Computing methodologies $\rightarrow$ Machine learning;

\section{KEYWORDS}

Multiobjective Optimization, Tree-structured Parzen Estimator, Computationally Expensive Optimization, Bayesian Optimization, Surrogate Modeling, Infill Criteria, Machine Learning

\section{ACM Reference Format:}

Yoshihiko Ozaki, Yuki Tanigaki, Shuhei Watanabe, and Masaki Onishi. 2020. Multiobjective Tree-structured Parzen Estimator for Computationally Expensive Optimization Problems. In Genetic and Evolutionary Computation Conference (GECCO '20), fuly 8-12, 2020, Cancún, Mexico. ACM, New York, NY, USA, 9 pages. https://doi.org/10.1145/3377930.3389817

Permission to make digital or hard copies of part or all of this work for personal or classroom use is granted without fee provided that copies are not made or distributed for profit or commercial advantage and that copies bear this notice and the full citation on the first page. Copyrights for third-party components of this work must be honored. For all other uses, contact the owner/author(s).

GECCO '20, fuly 8-12, 2020, Cancún, Mexico

(C) 2020 Copyright held by the owner/author(s)

ACM ISBN 978-1-4503-7128-5/20/07.

https://doi.org/10.1145/3377930.3389817

\section{INTRODUCTION}

In this paper, we address the following multiobjective optimization problem (MOP):

$$
\begin{array}{ll}
\text { minimize } & f(\boldsymbol{x})=\left(f_{1}(\boldsymbol{x}), \ldots, f_{m}(\boldsymbol{x})\right) \\
\text { subject to } & \boldsymbol{x} \in X
\end{array}
$$

where $X=X_{1} \times \cdots \times X_{n}$ is an $n$-dimensional decision space, and $f_{i}: X \rightarrow \mathbb{R}(i=1, \ldots, m)$ is an objective function. Typically, these objectives conflict against each other. Here the goal is to obtain a set of Pareto optimal solutions with a given evaluation budget. Important real-world problems are formulated in this manner, and such real-world problems are typically found to be computationally expensive. One example is the design of a tractor's air intake ventilation system [10]. This problem requires expensive computational fluid dynamics simulations to evaluate the objectives. Another example is Neural Architecture Search (NAS) that aims to identify a deep neural network (DNN) architecture with high performance and low computational cost [20]. This problem requires time-consuming DNN training to evaluate the objectives.

Objective functions of real-world problems, such as those mentioned above, are not time-efficient and in practice, evaluating them many times is impossible. Therefore, when considering real-world applications, using popular multiobjective optimization algorithms, such as NSGA-II [12] and MOEA/D [28], that require thousands of evaluations to converge may not be an appropriate choice for practitioners. This calls for developing an efficient algorithm to solve computationally expensive MOPs.

Tree-structured Parzen Estimator. The tree-structured Parzen estimator (TPE) [3, 5] is a single-objective Bayesian optimization algorithm for the hyperparameter optimization (HPO) of machine learning algorithms and NAS. A distinctive feature of this method is that it utilizes tree-structured adaptive Parzen estimators as a surrogate, whereas the standard Bayesian optimization algorithm utilizes Kriging (i.e., Gaussian process regression [27]). This surrogate naturally handles not only continuous variables but also discrete, categorical, and conditional variables that are difficult to handle using Kriging. Furthermore, this surrogate has lower computational complexity than Kriging and can scale to tens of variables and thousands of observations. TPE has been adopted as the standard solver of Hyperopt [4] and Optuna [1] - HPO frameworks that are widely used in the machine learning community. This method 
has also been successfully applied to tuning the hyperparameters of CMA-ES [29].

The success of TPE in expensive optimization problems indicates that it may outperform existing methods in MOPs in the case of a limited budget. Therefore, in this study, we extend TPE to a multiobjective optimization method, as discussed in Section 2.

Algorithms for Computationally Expensive MOPs. Several algorithms focusing on computationally expensive MOPs have been investigated, e.g., surrogate-assisted evolutionary algorithms (SAEAs) [9] and evolutionary multitasking optimization [14]. Currently, SAEAs are the most promising approach [8,11, 16, 19, 23, 24]. These algorithms accelerate optimization by combining an evolutionary algorithm with a cheap-to-evaluate surrogate. Knowles proposed ParEGO [19], which is an extension of the single-objective efficient global optimization (EGO) algorithm [18]. ParEGO reduces a MOP into a single-objective optimization problem via an augmented Tchebycheff aggregation. It utilizes the design and analysis of computer experiments (DACE) model [25] based on Kriging to approximate the objective landscape and evaluates the candidate solution with the maximum expected improvement (EI) value. Ponweiser et al. proposed SMS-EGO [24], which is another extension of the EGO algorithm. SMS-EGO also utilizes the DACE model; however, it differs from ParEGO in that the candidate is determined based on the hypervolume contribution in each iteration. Recently, Chugh et al. proposed K-RVEA [8, 11], which extends RVEA [7]. K-RVEA combines RVEA with Kriging and is especially effective for many-objective optimization problems. This method has been successful in the air intake ventilation system design [10]. Although Kriging is currently the most commonly used surrogate, several SAEAs employ surrogates other than Kriging. For example, Pan et al. proposed CSEA [23], which employs a feedforward neural network classifier that predicts the dominance relationship between candidate solutions and reference solutions, and Guo et al. proposed HeE-MOEA [16], which employs an ensemble of radial basis function networks and least square support vector machines. These surrogates are more scalable to increasing numbers of training samples and the dimension of the search space than Kriging.

Thus far, comparisons between SAEA, which is the mainstream of the approach for solving computationally expensive MOPs, and an alternative approach such as the method that we propose have not been well investigated.

Contributions. The contributions of this study have been summarized below.

- We propose an algorithm for computationally expensive MOPs called Multiobjective Tree-structured Parzen Estimator (MOTPE), which is an extension of TPE for multiobjective optimization.

- We perform comparisons between SAEAs and the proposed method. The empirical results demonstrate that in most cases, MOTPE approximates the Pareto front more effectively than existing methods with a limited evaluation budget.

- We discuss how the configuration of MOTPE affects both its behavior and performance.

\section{MULTIOBJECTIVE TREE-STRUCTURED PARZEN ESTIMATOR (MOTPE)}

In this section, we first provide mathematical preliminaries. Then, a brief explanation of TPE is offered. Finally, the proposed MOTPE algorithm is introduced.

\subsection{Preliminaries}

The following definitions and notations are used in this paper.

Definition 2.1. Vector $\boldsymbol{y}$ dominates vector $\boldsymbol{y}^{\prime}$ iff $\forall i: y_{i} \leq y_{i}^{\prime}$ and $\boldsymbol{y} \neq \boldsymbol{y}^{\prime}$, denoted $\boldsymbol{y}>\boldsymbol{y}^{\prime}$. Vector $\boldsymbol{y}$ weakly dominates vector $\boldsymbol{y}^{\prime}$ iff $\forall i: y_{i} \leq y_{i}^{\prime}$, denoted $\boldsymbol{y} \geq \boldsymbol{y}^{\prime}$.

Definition 2.2. Vector $\boldsymbol{y}$ and $\boldsymbol{y}^{\prime}$ are incomparable iff neither $\boldsymbol{y} \geq$ $\boldsymbol{y}^{\prime}$ nor $\boldsymbol{y}^{\prime} \geq \boldsymbol{y}$, denoted $\boldsymbol{y} \| \boldsymbol{y}^{\prime}$.

Definition 2.3. For a set of vectors $Y$ and a vector $\boldsymbol{y}$, define $Y>\boldsymbol{y}$ (resp. $Y \geq \boldsymbol{y}$ ) iff $\exists \boldsymbol{y}^{\prime} \in Y: \boldsymbol{y}^{\prime}>\boldsymbol{y}$ (resp. $\boldsymbol{y}^{\prime} \geq \boldsymbol{y}$ ). For a set of vectors $Y$ and a vector $\boldsymbol{y}$, also define $\boldsymbol{y}>Y$ (resp. $\boldsymbol{y} \geq Y$ ) iff $\exists \boldsymbol{y}^{\prime} \in Y: \boldsymbol{y}>\boldsymbol{y}^{\prime}$ (resp. $\boldsymbol{y} \geq \boldsymbol{y}^{\prime}$ ).

Definition 2.4. For a set of vectors $Y$ and a vector $\boldsymbol{y}$, define $Y \| \boldsymbol{y}$ (also denoted $\boldsymbol{y} \| Y$ ) iff $\forall \boldsymbol{y}^{\prime} \in Y: \boldsymbol{y} \| \boldsymbol{y}^{\prime}$.

Definition 2.5. For a set of vectors $Y$, the nondomination rank [12] of a vector $\boldsymbol{y} \in Y$ denoted $\operatorname{rank}(\boldsymbol{y}) \in \mathbb{N}$ is defined as follows:

- $\operatorname{rank}(\boldsymbol{y})=1$ iff $\nexists \boldsymbol{y}^{\prime} \in Y: \boldsymbol{y}^{\prime}>\boldsymbol{y}$.

- $\forall k \geq 1: \operatorname{rank}(\boldsymbol{y})=k+1$ iff $\nexists \boldsymbol{y}^{\prime} \in Y: \boldsymbol{y}^{\prime}>\boldsymbol{y} \wedge \boldsymbol{y}^{\prime} \notin$ $\bigcup_{i \leq k}\left\{\boldsymbol{y}^{\prime} \in Y \mid \operatorname{rank}\left(\boldsymbol{y}^{\prime}\right)=i\right\}$.

We also denote by $Y_{\operatorname{rank}(k)}$ the set $\{\boldsymbol{y} \in Y \mid \operatorname{rank}(\boldsymbol{y})=k\}$. Vector $\boldsymbol{y} \in Y$ is nondominated iff $\operatorname{rank}(\boldsymbol{y})=1$.

Definition 2.6. The hypervolume indicator [6] $I_{\mathrm{H}}$ of a set of vectors $Y$ can be defined as the hypervolume of the space that is dominated by set $Y$ and bounded by a reference point $r$ :

$$
I_{\mathrm{H}}(Y):=\lambda\left(\bigcup_{\boldsymbol{y} \in Y}\left[f_{1}(\boldsymbol{y}), r_{1}\right] \times \cdots \times\left[f_{m}(\boldsymbol{y}), r_{m}\right]\right)
$$

where $\lambda(S)$ is the Lebesgue measure of a set $S$, and $\left[f_{1}(\boldsymbol{y}), r_{1}\right] \times$ $\cdots \times\left[f_{m}(\boldsymbol{y}), r_{m}\right]$ is the $m$-dimensional hypercuboid consisting of all vectors that are weakly dominated by vector $\boldsymbol{y}$ but not weakly dominated by the reference point.

\subsection{Overview of TPE}

Before introducing MOTPE, we outline TPE for single-objective minimization [3, 5] (Algorithm 1).

Assume a set of observations that takes $\left\{\left(x^{(1)}, y^{(1)}\right), \ldots\right.$, $\left.\left(\boldsymbol{x}^{(k)}, y^{(k)}\right)\right\}$. TPE defines $p(\boldsymbol{x} \mid y)$ using the following two probability density functions:

$$
p(\boldsymbol{x} \mid y)= \begin{cases}l(\boldsymbol{x}) & \text { if } y^{*}>y \\ g(\boldsymbol{x}) & \text { if } y \geq y^{*}\end{cases}
$$

where $l(x)$ is the probability density function formed using the observed variables $\left\{\boldsymbol{x}^{(i)}\right\}$ such that $y^{*}>y^{(i)}\left(=f\left(\boldsymbol{x}^{(i)}\right)\right)$, and $g(\boldsymbol{x})$ is the probability density function using the remaining observations. The value $y^{*}$ is selected to be a quantile $y$ of the observed $y$ values satisfying $p\left(y^{*}>y\right)=\gamma$. Briefly, $l(\boldsymbol{x})$ models the density 

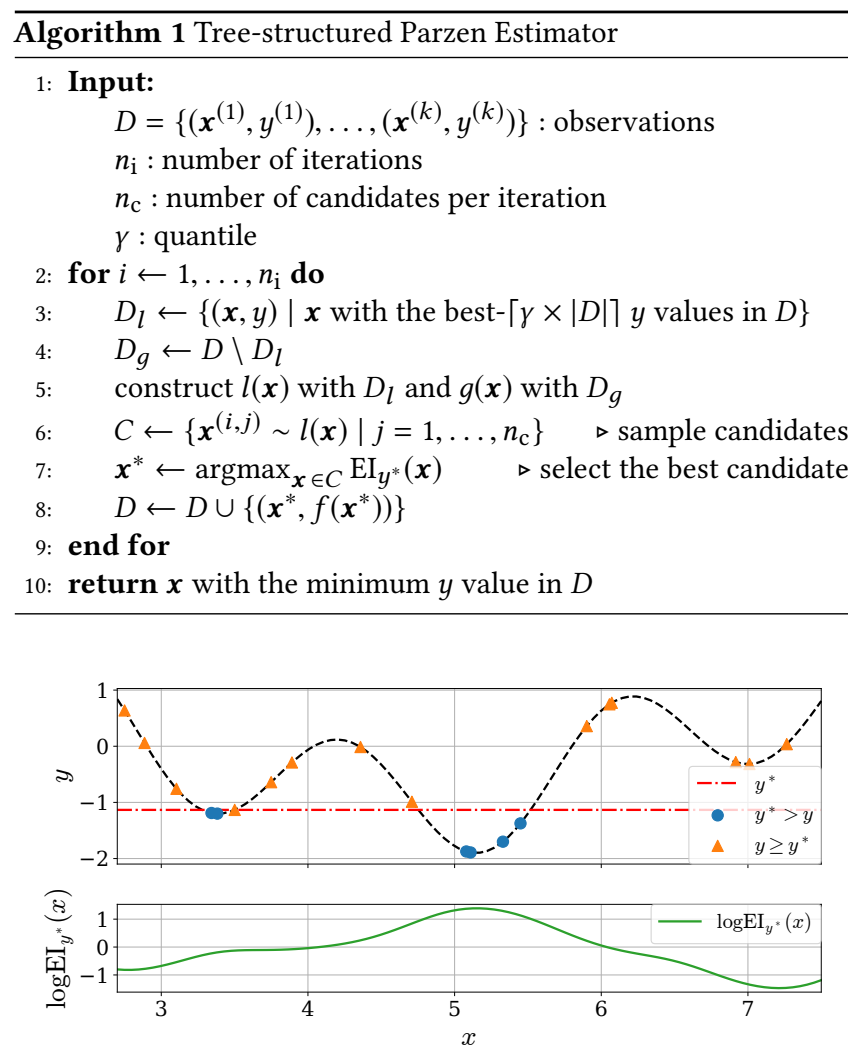

Figure 1: Example plot of $\log \mathrm{EI}_{y^{*}}(x)$ for $y=\sin (x)+\sin \left(\frac{10}{3} x\right)$ (black dotted line) in the range $[2.7,7.5]$ with several observations marked with a “•” or “ $\Delta$. . Here, $\operatorname{argmax}_{x} \mathrm{EI}_{y^{*}}(x)$ $\left(=\operatorname{argmax}_{x} \log \mathrm{EI}_{y^{*}}(x)\right)$ appears to be preferable.

of better observations, and $g(\boldsymbol{x})$ models the density of poor observations. These models are tree-structured hierarchical processes constructed using adaptive Parzen estimators as detailed in the previous papers [3, 5]. In lines 3-4 of Algorithm 1, the observations are split into $D_{l}$ and $D_{g}$ to construct the models, and this can be easily achieved by sorting the observations based on the $y$ values.

TPE employs the following EI function as the infill criterion (i.e., acquisition function) [3]:

$$
\begin{aligned}
\mathrm{EI}_{y^{*}}(\boldsymbol{x}) & :=\int_{-\infty}^{\infty} \max \left(y^{*}-y, 0\right) p(y \mid \boldsymbol{x}) d y \\
& =\int_{-\infty}^{y^{*}}\left(y^{*}-y\right) p(y \mid \boldsymbol{x}) d y \\
& \propto\left(\gamma+(1-\gamma) \frac{g(\boldsymbol{x})}{l(\boldsymbol{x})}\right)^{-1} .
\end{aligned}
$$

In lines 6-7 of Algorithm 1, several candidates are sampled from $l(\boldsymbol{x})$ and the best candidate $\boldsymbol{x}^{*}$ with the greatest EI value is selected in each iteration ${ }^{1}$. Figure 1 shows an example of $\log \mathrm{EI}_{y^{*}}$ for a benchmark function.

\footnotetext{
${ }^{1}$ Although this is the standard procedure, generally, any optimization method to maximize EI can be employed. This is also true for the candidate selection step of MOTPE (Algorithm 3, lines 5-6).
}

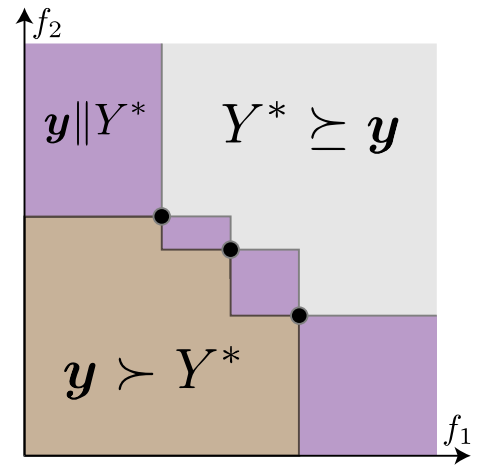

Figure 2: Areas are filled with different colors based on the relationship between $y$ and $Y^{*}$. Black points belong to $Y^{*}$.

\subsection{TPE for MOPs}

In this subsection, we introduce the MOTPE algorithm, which is an extension of TPE for multiobjective optimization.

Assume a set of observations that takes $\left\{\left(\boldsymbol{x}^{(1)}, \boldsymbol{y}^{(1)}\right), \ldots\right.$, $\left.\left(\boldsymbol{x}^{(k)}, \boldsymbol{y}^{(k)}\right)\right\}$. MOTPE defines $p(\boldsymbol{x} \mid \boldsymbol{y})$ using the following two probability density functions:

$$
p(\boldsymbol{x} \mid \boldsymbol{y})= \begin{cases}l(\boldsymbol{x}) & \text { if } \boldsymbol{y}>Y^{*} \cup \boldsymbol{y} \| Y^{*} \\ g(\boldsymbol{x}) & \text { if } Y^{*} \geq \boldsymbol{y}\end{cases}
$$

where $Y^{*}$ is a set of objective vectors such that $p\left(\boldsymbol{y}>Y^{*} \cup \boldsymbol{y} \| Y^{*}\right)=\gamma$. In addition, $l(\boldsymbol{x})$ is the probability density function formed using the observed decision vectors $\left\{\boldsymbol{x}^{(i)}\right\}$ such that $\boldsymbol{y}^{(i)}\left(=\boldsymbol{f}\left(\boldsymbol{x}^{(i)}\right)\right)>$ $Y^{*}$ or $\boldsymbol{y}^{(i)} \| Y^{*}$, and $g(\boldsymbol{x})$ is the probability density function using the remaining observations. Figure 2 illustrates the relationship between $\boldsymbol{y}$ and $Y^{*}$ in the two-dimensional case. MOTPE constructs the model in an identical manner to the original TPE algorithm.

Greedy Splitting of the Observations. MOTPE orders a sophisticated algorithm to split observations including many incomparable ones. The MOTPE algorithm theoretically utilizes $Y^{*}$ such that $p\left(\boldsymbol{y}>Y^{*} \cup \boldsymbol{y} \| Y^{*}\right)=\gamma$ to split the observations into subsets for $l(\boldsymbol{x})$ and for $g(\boldsymbol{x})$. However, in practice, the observations are directly split for a specific $\gamma$ in a greedy manner, as described in Algorithm 2. Algorithm 2 comprises two steps. The first step (lines 4-7) greedily appends better nondomination ranked observations to the largest extent possible to $D_{l}$. The second step (line 8) appends the set obtained as a result of the hypervolume subset selection problem (HSSP) [2] to $D_{l}$. HSSP is a problem finding a subset of a specific size that maximizes the hypervolume indicator with a given reference point. HSSP is known to be a submodular maximization; thus, it is possible to obtain a $1-1 / e$ approximation via the greedy method [15, 22]. The subroutine SOLVE_HSSP $(S, s)$ returns the result of HSSP for a set $S$ and the size of subset $s$. The vector $\left(1.1 \times \max _{\boldsymbol{y} \in D_{\boldsymbol{y}}} y_{1}, \ldots, 1.1 \times \max _{\boldsymbol{y} \in D_{\boldsymbol{y}}} y_{m}\right)$, where $D_{\boldsymbol{y}}=\left\{\boldsymbol{y} \mid(\boldsymbol{x}, \boldsymbol{y}) \in D_{\operatorname{rank}(i)}\right\}$, is used as the reference point for HSSP in our experiments.

Properly setting $\gamma$ is important for the performance of MOTPE, and in Section 3.2, we describe the effect of $\gamma$ on the behavior of the method based on empirical data. 


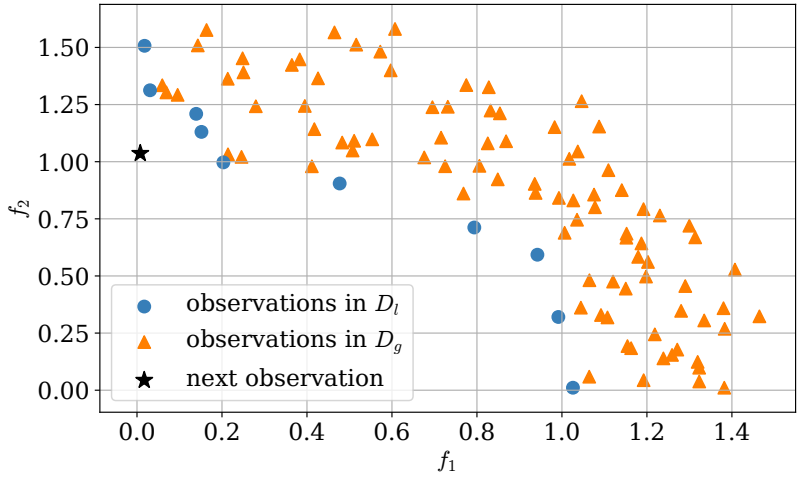

(a) DTLZ2: $\gamma=0.1,|D|=100$

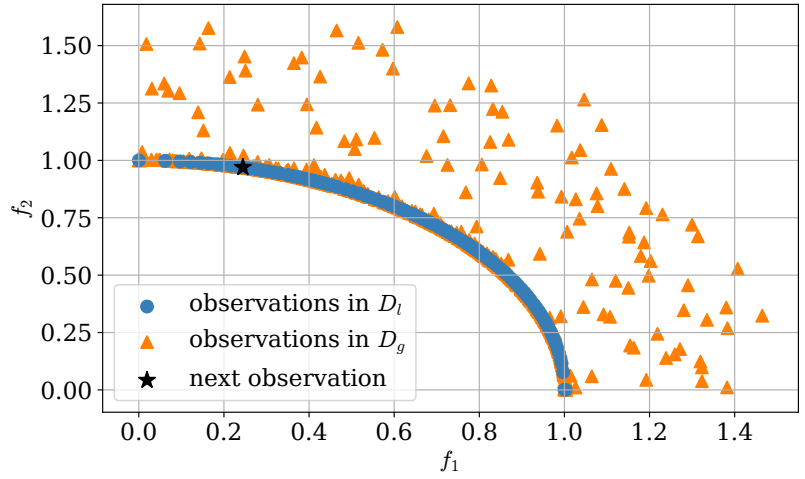

(b) DTLZ2: $\gamma=0.1,|D|=1000$

Figure 3: Visualizations examples of MOTPE in the objective space.

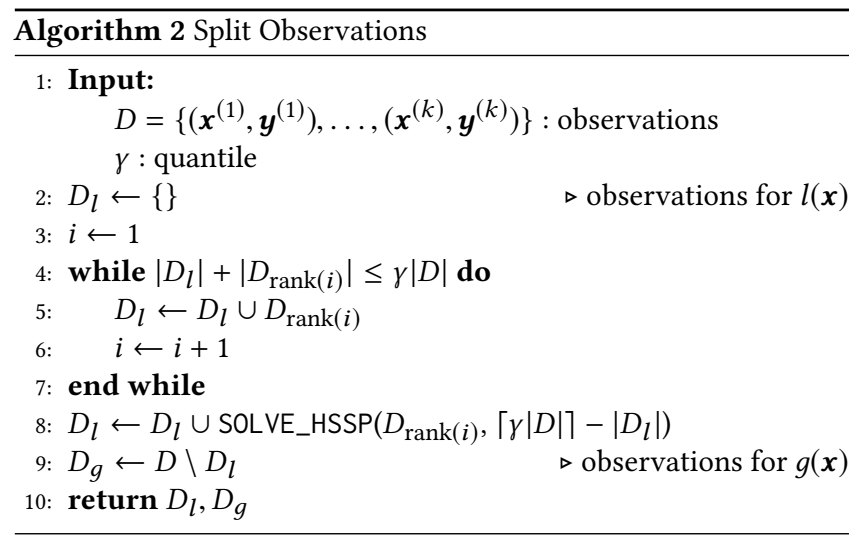

Calculation of the Expected Hypervolume Improvement. In MOTPE, we substitute the expected hypervolume improvement (EHVI) for EI in TPE to select a candidate $\boldsymbol{x}^{*}$ to evaluate. Here, EHVI for $Y^{*}$ and $\boldsymbol{x}$ is calculated as follows:

$$
\begin{aligned}
\operatorname{EHVI}_{Y^{*}}(\boldsymbol{x}) & =\int_{R}\left(I_{\mathrm{H}}\left(Y^{*} \cup\{\boldsymbol{y}\}\right)-I_{\mathrm{H}}\left(Y^{*}\right)\right) p(\boldsymbol{y} \mid \boldsymbol{x}) d \boldsymbol{y} \\
& =\int_{R}\left(I_{\mathrm{H}}\left(Y^{*} \cup\{\boldsymbol{y}\}\right)-I_{\mathrm{H}}\left(Y^{*}\right)\right) \frac{p(\boldsymbol{x} \mid \boldsymbol{y}) p(\boldsymbol{y})}{p(\boldsymbol{x})} d \boldsymbol{y},
\end{aligned}
$$

where $R=\left\{\boldsymbol{y} \mid \boldsymbol{y}>Y^{*} \cup \boldsymbol{y} \| Y^{*}\right\}$. Based on Equation (5), $\gamma=p(\boldsymbol{y} \in$ $R)$, and $p(\boldsymbol{x})=\int p(\boldsymbol{x} \mid \boldsymbol{y}) p(\boldsymbol{y}) d \boldsymbol{y}=\gamma l(\boldsymbol{x})+(1-\gamma) g(\boldsymbol{x})$; therefore, the numerator of $\operatorname{EHVI}_{Y^{*}}(\boldsymbol{x})$ is

$$
\begin{aligned}
& \int_{R}\left(I_{\mathrm{H}}\left(Y^{*} \cup\{\boldsymbol{y}\}\right)-I_{\mathrm{H}}\left(Y^{*}\right)\right) p(\boldsymbol{x} \mid \boldsymbol{y}) p(\boldsymbol{y}) d \boldsymbol{y} \\
= & l(\boldsymbol{x}) \underbrace{\int_{R}\left(I_{\mathrm{H}}\left(Y^{*} \cup\{\boldsymbol{y}\}\right)-I_{\mathrm{H}}\left(Y^{*}\right)\right) p(\boldsymbol{y}) d \boldsymbol{y}}_{=C_{Y^{*}}(\text { constant w.r.t. } \boldsymbol{x})} \\
= & C_{Y^{*}} l(\boldsymbol{x}),
\end{aligned}
$$

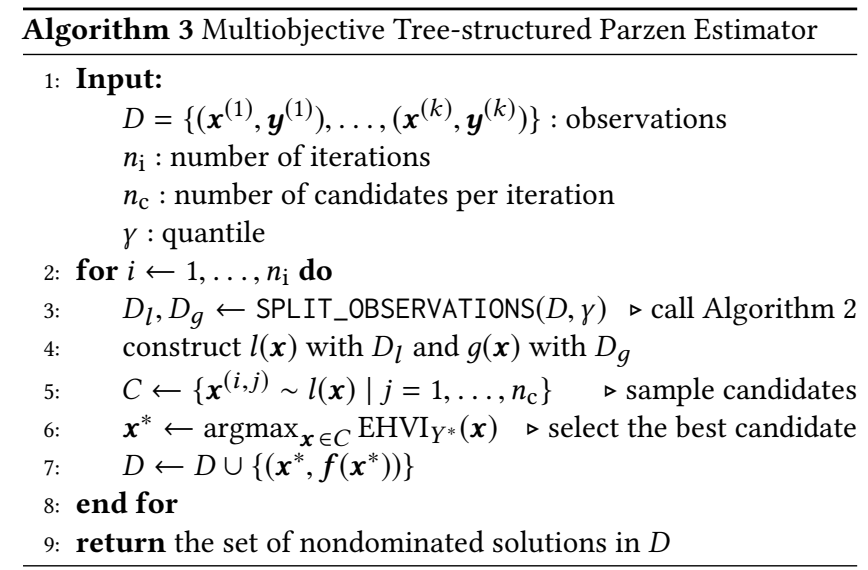

and finally, we obtain the following:

$$
\begin{aligned}
\operatorname{EHVI}_{Y^{*}}(\boldsymbol{x}) & =\frac{C_{Y^{*}} l(\boldsymbol{x})}{p(\boldsymbol{x})} \\
& =\frac{C_{Y^{*}} l(\boldsymbol{x})}{\gamma l(\boldsymbol{x})+(1-\gamma) g(\boldsymbol{x})} \\
& =\frac{C_{Y^{*}}}{\gamma+(1-\gamma) \frac{g(\boldsymbol{x})}{l(\boldsymbol{x})}} \\
& \propto\left(\gamma+(1-\gamma) \frac{g(\boldsymbol{x})}{l(\boldsymbol{x})}\right)^{-1} .
\end{aligned}
$$

In each iteration, the algorithm selects the candidate $\boldsymbol{x}^{*}$ with the greatest EHVI. This $x^{*}$ can be easily obtained by maximizing $l(\boldsymbol{x}) / g(\boldsymbol{x})$ based on the theoretical result in Equation (8).

The pseudocode of MOTPE is provided in Algorithm 3. MOTPE inherits the properties of TPE, which allow it to handle continuous, discrete, categorical, and conditional variables naturally, and evaluate candidate solutions in parallel asynchronously [3]. Figure 3 illustrates that MOTPE successfully approximates the Pareto front of the DTLZ2 benchmark problem [13]. 
Table 1: Experimental results (§ 3.1): mean hypervolume \pm standard deviation. $m$ is the number of objectives, $n$ is the number of variables, and $k$ and $l$ are the working parameters defined in the benchmark problems. The results of SMS-EGO are available only in the case of $m=2$ because the required computation time was too high in the case of $m=4$. Here, the best value is highlighted for each problem, and “ $\approx$ ” (resp. “-”, “+”) indicates that the result is equivalent to (resp. worse than, better than) the MOTPE result based on the Wilcoxon rank sum test at the 0.05 significance level.

\begin{tabular}{c|c|cc|c|cc|cc}
\hline Problem (m, n, k, l) & MOTPE & \multicolumn{2}{|c|}{ ParEGO } & \multicolumn{2}{|c|}{ SMS-EGO } & \multicolumn{2}{|c}{ K-RVEA } \\
\hline WFG4 (2, 9, 1, 8) & $\mathbf{7 . 7 3} \pm \mathbf{0 . 1 0}$ & $6.52 \pm 0.16$ & $(-)$ & $6.83 \pm 0.20$ & $(-)$ & $7.25 \pm 0.13$ & $(-)$ \\
WFG5 (2, 9, 1, 8) & $7.18 \pm 0.20$ & $\mathbf{7 . 9 7} \pm \mathbf{0 . 0 5}$ & $(+)$ & $7.76 \pm 0.20$ & $(+)$ & $7.31 \pm 0.46$ & $(+)$ \\
WFG6 (2, 9, 1, 8) & $\mathbf{6 . 9 3} \pm \mathbf{0 . 2 6}$ & $6.24 \pm 0.18$ & $(-)$ & $6.00 \pm 0.31$ & $(-)$ & $5.93 \pm 0.36$ & $(-)$ \\
WFG7 (2, 9, 1, 8) & $\mathbf{7 . 4 5} \pm \mathbf{0 . 2 5}$ & $6.00 \pm 0.22$ & $(-)$ & $6.12 \pm 0.23$ & $(-)$ & $6.65 \pm 0.27$ & $(-)$ \\
WFG8 (2, 9, 1, 8) & $\mathbf{6 . 1 0} \pm \mathbf{0 . 2 4}$ & $4.91 \pm 0.18$ & $(-)$ & $5.65 \pm 0.34$ & $(-)$ & $6.02 \pm 0.15$ & $(\approx)$ \\
WFG9 (2, 9, 1, 8) & $\mathbf{7 . 3 4} \pm \mathbf{0 . 4 5}$ & $5.96 \pm 0.29$ & $(-)$ & $6.07 \pm 0.33$ & $(-)$ & $6.23 \pm 0.39$ & $(-)$ \\
\hline WFG4 (4, 9, 3, 6) & $\mathbf{6 2 0 . 4 2} \pm \mathbf{3 7 . 9 7}$ & $464.87 \pm 20.41$ & $(-)$ & - & - & $593.25 \pm 17.41$ & $(-)$ \\
WFG5 (4, 9, 3, 6) & $\mathbf{6 0 6 . 9 3 \pm 1 5 . 6 5}$ & $\mathbf{6 4 2 . 4 3 \pm 2 1 . 8 9}$ & $(+)$ & - & - & $602.98 \pm 24.07$ & $(\approx)$ \\
WFG6 (4, 9, 3, 6) & $\mathbf{5 7 0 . 8 3} \pm \mathbf{3 7 . 5 2}$ & $529.22 \pm 9.90$ & $(-)$ & - & - & $471.43 \pm 35.14$ & $(-)$ \\
WFG7 (4, 9, 3, 6) & $\mathbf{6 3 0 . 6 9 \pm 2 4 . 3 1}$ & $425.65 \pm 19.52$ & $(-)$ & - & - & $561.23 \pm 17.88$ & $(-)$ \\
WFG8 (4, 9, 3, 6) & $\mathbf{4 5 6 . 6 6} \pm \mathbf{2 1 . 0 2}$ & $348.58 \pm 8.67$ & $(-)$ & - & - & $413.36 \pm 15.08$ & $(-)$ \\
WFG9 (4, 9, 3, 6) & $\mathbf{5 9 5 . 2 9} \pm \mathbf{2 5 . 5 6}$ & $371.87 \pm 22.24$ & $(-)$ & - & - & $486.08 \pm 41.79$ & $(-)$ \\
\hline
\end{tabular}

\section{NUMERICAL RESULTS}

In this section, we describe two experiments that were conducted and discuss the results. First, we compare MOTPE with existing methods on several benchmark problems. We then empirically investigate the optimal setting for the quantile and the relationship between the quantile setting and the proposed method's performance.

\subsection{Comparisons with Other Methods}

We compared MOTPE to ParEGO [19], SMS-EGO [24], and KRVEA [8], which are well-known SAEAs. These three methods employ Kriging-based surrogates, whereas the proposed MOTPE employs tree-structured adaptive Parzen estimators. The implementations of these methods are provided in PlatEMO [26], which is an evolutionary multiobjective optimization platform. The Python implementation of MOTPE is available on our GitHub repository ${ }^{2}$.

Experimental Setup. The WFG4-9 benchmark problems [17] were used in our experiments, and we prepared two settings, i.e., ( $m=2, n=9, k=1, l=8)$ and $(m=4, n=9, k=3, l=6)$, where $m$ is the number of objectives, $n$ is the number of variables, and $k$ and $l$ are the working parameters defined in the benchmark problems. We consider that these settings are reasonable because the numbers of objectives and variables in the target real-world problems are also relatively small, e.g., $m$ and $n$ are 3 and 4, respectively, in the literature [10]. Here, to simulate computationally expensive objectives that cannot be evaluated many times in practice, the evaluation budget (including initial evaluations) was set to 250 for all compared algorithms. The number of initial observations was set to $11 n-1$, and the initial solutions were sampled using Latin hypercube sampling (LHS) [21]. This initialization procedure has been used frequently in existing studies $[8,19,24]$. The number of iterations $n_{\mathrm{i}}$ was set to $250-(11 n-1)$. Relative to the MOTPE

\footnotetext{
${ }^{2}$ https://github.com/y0z/motpe.
}

parameters, we set $\gamma=0.10$, which is the default in Optuna [1], and $n_{\mathrm{c}}=24$ which is the default in both HyperOpt [4] and Optuna. Note that the ParEGO, SMS-EGO, and K-RVEA settings were set to the PlatEMO defaults. For each method and setting, 21 optimization runs were performed. As a metric of performance, the hypervolume indicator with the reference point $(3,5)$ (resp. $(3,5,7,9))$ for $m=2$ (resp. $m=4$ ) was used.

Results and Discussion. Table 1 shows the experimental results. Here, the best result for each benchmark problem is highlighted. The Wilcoxon rank sum test was performed to compare the results obtained by the proposed MOTPE and the other algorithms at the 0.05 significance level. As achieving the best in 10 out of the 12 benchmark problems, the proposed MOTPE algorithm outperformed the other three SAEAs: ParEGO, SMS-EGO, and K-RVEA. Moreover, the results show that the proposed method scales to at least four objectives without performance degradation. Here, K-RVEA was the overall second-best method because it obtained second-place results eight times. Note that ParEGO achieved the best results for WFG5. In addition, SMS-EGO was comparable to ParEGO for $m=2$; however, it was prohibitive for $m=4$, as its computation time was too high. The standard deviations of the results were relatively small and not differ significantly between the four methods. This indicates that the methods had stable performance and were not sensitive to initialization. Figure 4 shows the plots of the obtained Pareto front approximations obtained by each method for $m=2$. As can be seen, in most cases, the proposed MOTPE converged faster while maintaining sufficient diversity in the solutions. For WFG4-9, MOTPE did not outperform the compared methods for only WFG5. The result of MOTPE for WFG5 does not look very poor; however, the results obtained by the other methods look extremely good (Figure 4b). Perhaps, the WFG5 landscape may be easier to approximate than other benchmark problems for Kriging-based surrogates. 


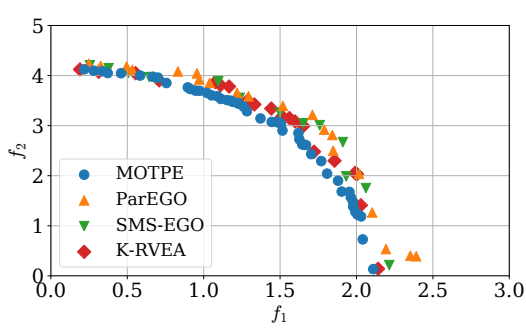

(a) WFG4 $(2,9,1,8)$

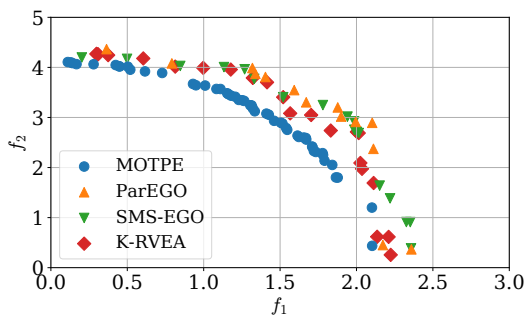

(d) WFG7 $(2,9,1,8)$

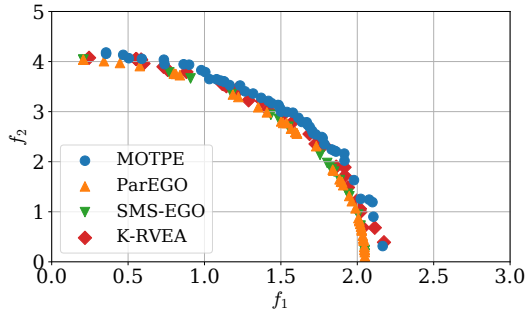

(b) WFG5 $(2,9,1,8)$

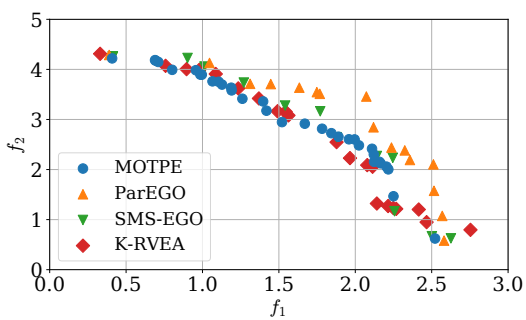

(e) WFG8 $(2,9,1,8)$

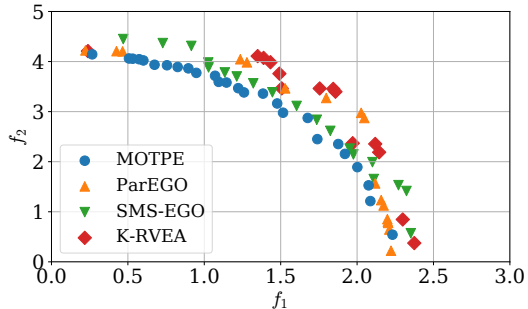

(c) WFG6 $(2,9,1,8)$

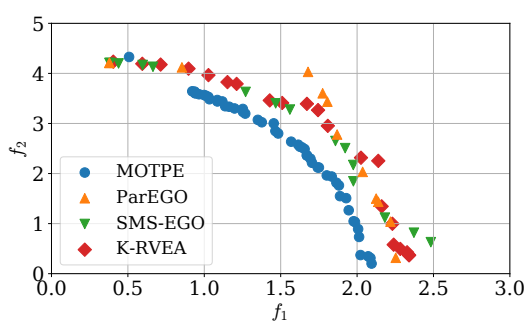

(f) WFG9 $(2,9,1,8)$

Figure 4: Plots of the Pareto front approximations (§ 3.1): the results with the median hypervolume values.

\subsection{Parameter Influence: Quantile $\gamma$}

The quantile $\gamma$ of MOTPE has a significant impact on constructing its surrogate. It is the most important parameter relative to optimization performance. Here, we empirically investigate the effects of $\gamma$ on the proposed method's behavior and identify the optimal setting for $\gamma$.

Experimental Setup. To investigate the influence of $\gamma$, MOTPE was tested on benchmark problems with different $\gamma$ settings. There were 20 settings in the experiment: $\gamma=0.01$ and $0.05,0.10, \ldots, 0.95$. The benchmark problems and remaining settings of the method were identical to those in Section 3.1. For each setting, 21 optimization runs were performed, and the hypervolume indicator was used as the performance metric with the reference point $(3,5)$ (resp. $(3,5,7,9))$ for $m=2$ (resp. $m=4)$.

Results and Discussion. Figure 5 presents plots of the mean hypervolumes for each $\gamma$ setting. The results indicate that $\gamma$ values between 0.05 and 0.10 were optimal for $m=2$, and $\gamma$ values between 0.10 and 0.25 were optimal for $m=4$. As can be seen, the obtained optimal $\gamma$ setting tends to be very small for $m=2$ and relatively large for $m=4$. These results also indicate that the performance of MOTPE is superior to that of the other methods for a variety of $\gamma$ settings. However, setting $\gamma$ to a value that is too small or too large causes serious performance degradation and should be avoided. Our empirical recommendation is $\gamma=0.10$, which is the intersection of the optimal $\gamma$ ranges for $m=2$ and $m=4$.

Now, we discuss the effect of $\gamma$ on the behavior of MOTPE. We consider the following four types of situations based on the relationship between $\gamma$ and the ratio of the nondominated observations to the number of observations, i.e., $r_{\mathrm{n}}=\left|D_{\operatorname{rank}(1)}\right| /|D|$ :
(1) $\gamma \ll r_{\mathrm{n}}$

(2) $\gamma \leq r_{\mathrm{n}}$.

(3) $\gamma>r_{\mathrm{n}}$.

(4) $\gamma \gg r_{\mathrm{n}}$.

In the situation (1), only a small subset of nondominated observations is used to construct $l(\boldsymbol{x})$, while the remaining observations are used to construct $g(\boldsymbol{x})$. As a result, candidates to evaluate sampled from $l(\boldsymbol{x})$ lack diversity. In addition, when $g(\boldsymbol{x})$ is constructed using many good observations, the EHVI criterion for selecting the best candidate becomes ineffective. As a result, the optimization performance of the method decreases. Figure 6 a illustrates the situation (1).

In the situation (2), a subset of nondominated observations is used to construct $l(\boldsymbol{x})$, while the remaining observations are used to construct $g(\boldsymbol{x})$. Typically, a set of observations with good diversity in the decision space has a large hypervolume. Therefore, such a set tends to be selected in the HSSP step of Algorithm 2. As a result, a smaller $\gamma$ results in MOTPE to using observations with high diversity to construct $l(\boldsymbol{x})$, and the method becomes more diversityoriented. Additionally, a small $\gamma$ also stabilizes the performance of the method. Figure 6b illustrates the situation (2).

In the situation (3), not only nondominated observations but also several dominated observations are used to construct $l(x)$, while the remaining observations are used to construct $g(\boldsymbol{x})$. Actively using the dominated observations can sometimes improve performance; however, using dominated observations usually slows down the convergence of the method. In addition, the method in this situation tends to converge to a poor result when the diversity of the solutions is poor because it has less pressure to improve diversity 


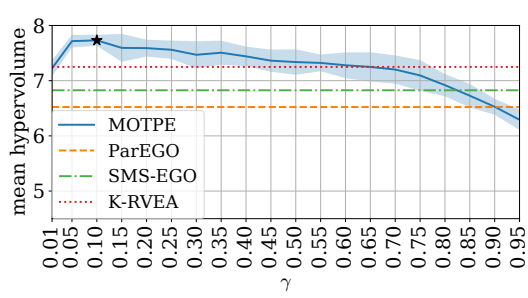

(a) WFG4 $(2,9,1,8)$

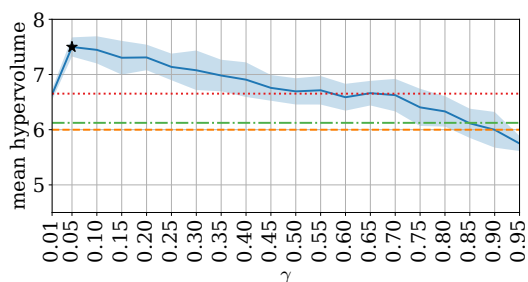

(d) WFG7 $(2,9,1,8)$

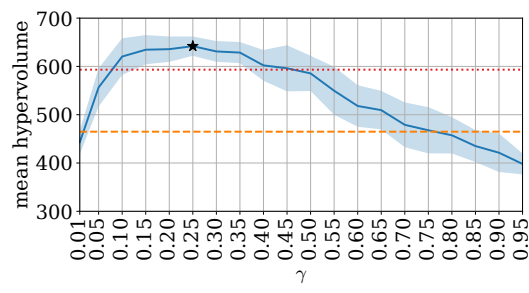

(g) WFG4 $(4,9,3,6)$

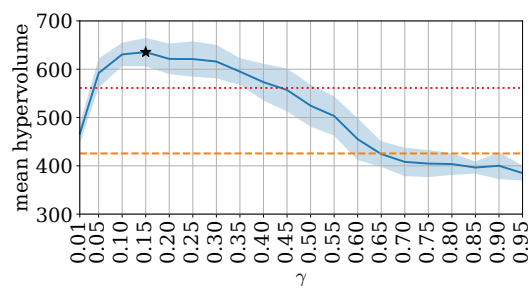

(j) WFG7 $(4,9,3,6)$

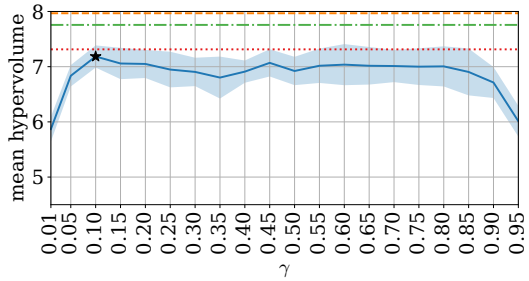

(b) WFG5 $(2,9,1,8)$

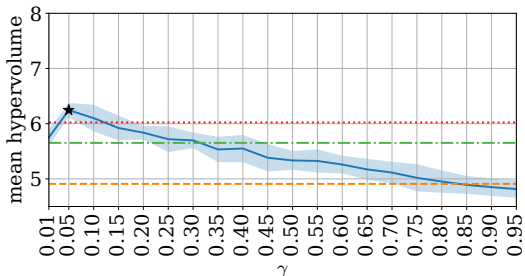

(e) WFG8 $(2,9,1,8)$

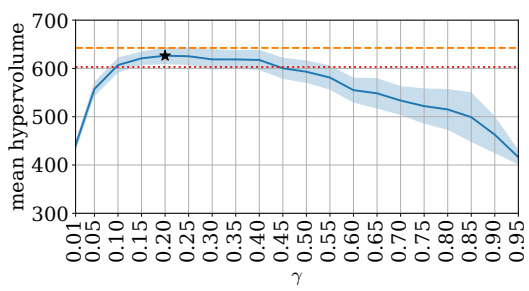

(h) WFG5 $(4,9,3,6)$

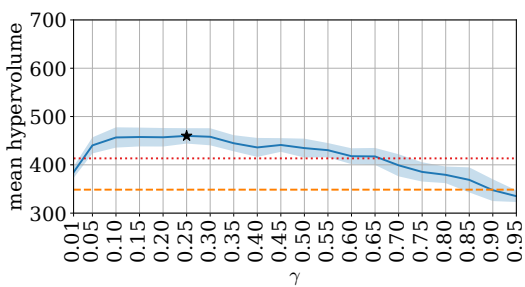

(k) WFG8 (4, 9, 3, 6)

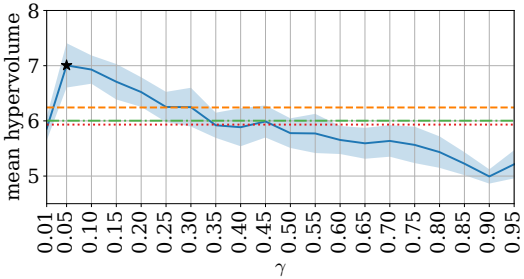

(c) WFG6 $(2,9,1,8)$

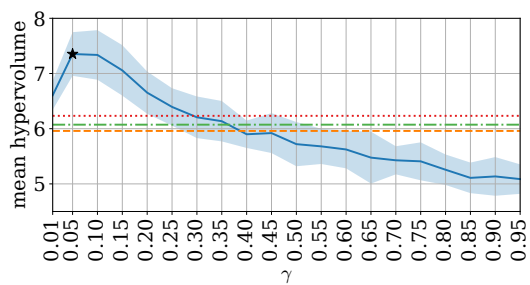

(f) WFG9 $(2,9,1,8)$

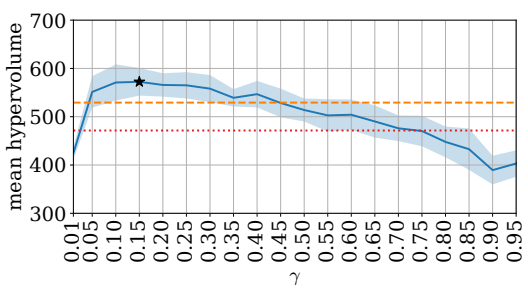

(i) WFG6 $(4,9,3,6)$

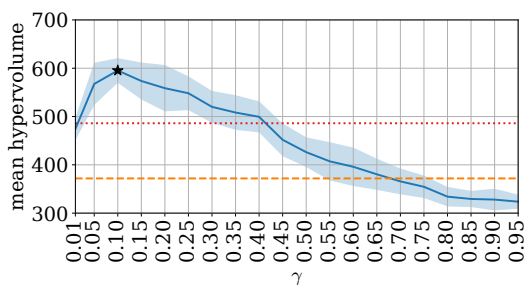

(l) WFG9 $(4,9,3,6)$

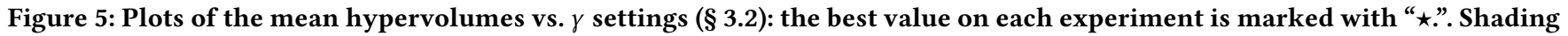
represents \pm standard deviation, and the dotted lines represent the results of the other methods listed in Table 1 . The legends in (b)-(l) are the same as in (a) and are omitted.

compared to the situation (2). The large standard deviations indicate the instability of this situation. Figure 6c illustrates the situation (3).

In the situation (4), not only good observations but also many poor observations are used to construct $l(x)$ while the remaining observations are used to construct $g(\boldsymbol{x})$. The poor observations prevent constructing a fine $l(\boldsymbol{x})$, and performance decreases because poor candidates are sampled. Figure 6d illustrates the situation (4).

Based on the above discussion, the situation (2) is the most preferable. MOTPE approximately controls the pressure to produce a diversity of solutions via $\gamma$ in the situation (2) while achieving good convergence. The optimal $\gamma$ setting for $m=4$ is larger than that for $m=2$ in our empirical results because nondominated solutions can be easily obtained in the case of $m=4$. This is generally true because the higher the dimension is, the more difficult it is to satisfy the condition $\forall i: y_{i} \leq y_{i}^{\prime}$ for two vectors $\boldsymbol{y}$ and $\boldsymbol{y}^{\prime}$.

To make $\gamma$ more robust to the objective dimension, $\gamma$ can be changed dynamically, i.e., by setting $\gamma=\alpha r_{\mathrm{n}}$ with a parameter $\alpha \in\left(0,1 / r_{\mathrm{n}}\right)$, during optimization rather than using a constant value. A self-adaptive $\gamma$ appears to be useful; however, the proper tuning of parameter $\alpha$ is still required. 


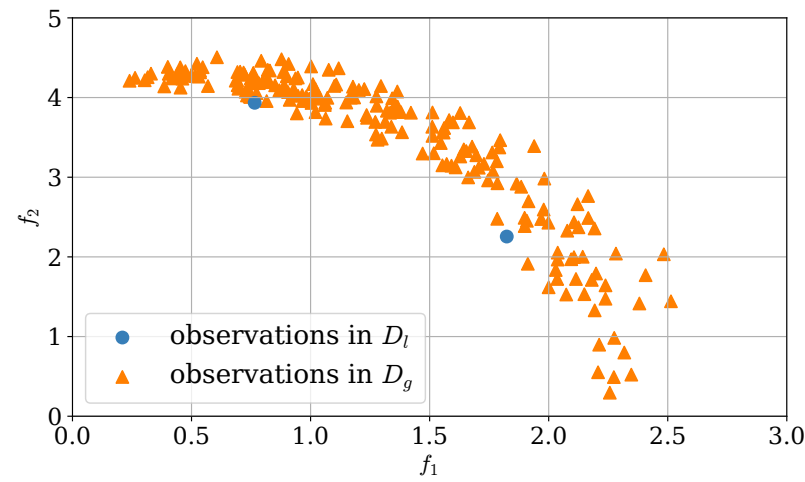

(a) WFG4 $(2,9,1,8): \gamma=0.01,|D|=200$

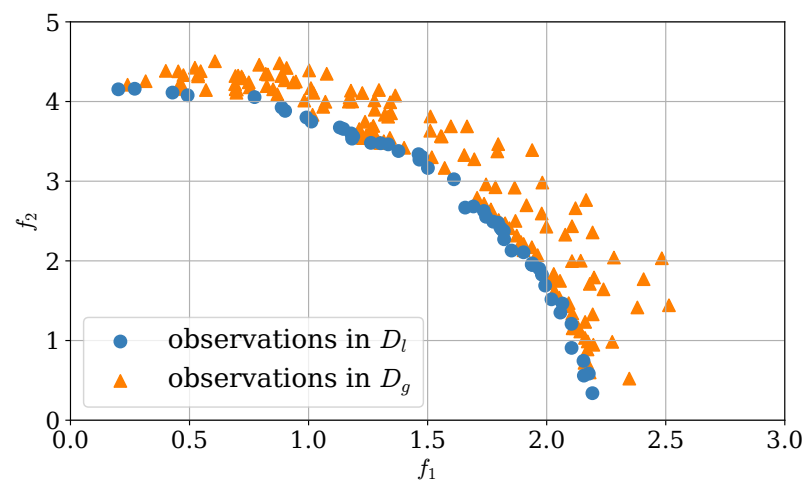

(c) WFG4 $(2,9,1,8): \gamma=0.25,|D|=200$

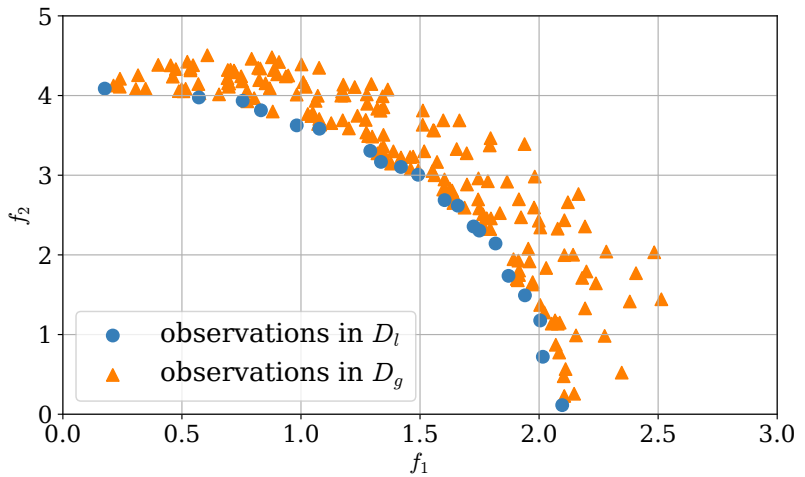

(b) WFG4 $(2,9,1,8): \gamma=0.10,|D|=200$

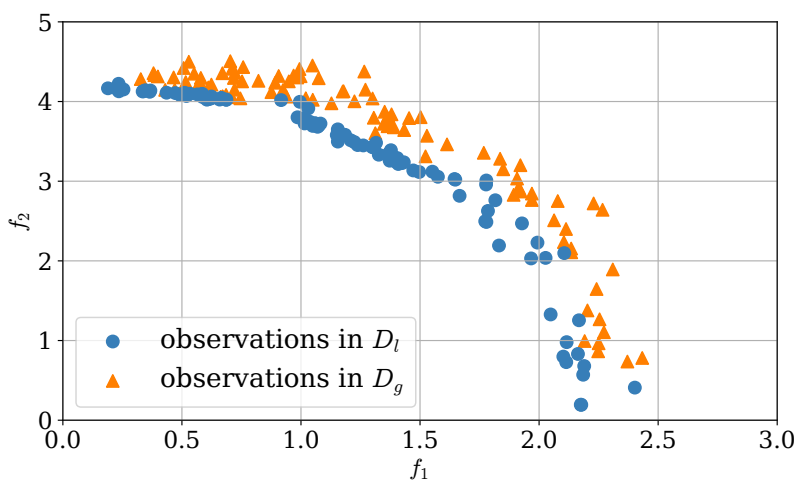

(d) WFG4 $(2,9,1,8): \gamma=0.50,|D|=200$

Figure 6: Visualization examples of MOTPE in the objective space with different $\gamma$ settings for the four situations.

\section{CONCLUDING REMARKS}

In this study, we extended TPE to multiobjective optimization to solve computationally expensive MOPs. Our empirical results demonstrate that, in most cases, the proposed MOTPE converges faster than existing methods while maintaining a sufficient diversity of solutions on several benchmark problems. These results prove that MOTPE is a potential solution to address computationally expensive MOPs, and to guarantee good performance for a set of real-world problems that are similar to the benchmark problems. We also empirically investigated the effects of the quantile parameter of the method. Our results demonstrate that this quantile parameter plays an important role in controlling the pressure to produce diversity.

In the future, we will perform further numerical experiments to verify the performance of the method on problems with more complex search spaces that include continuous, discrete, categorical, and conditional variables that frequently appear in important real-world problems, e.g. NAS, and are difficult to handle using Kriging-based surrogates. Then, we will apply MOTPE to realworld problems to demonstrate its capability and performance in practical applications.

\section{ACKNOWLEDGMENTS}

This paper is based on results obtained from a project commissioned by the New Energy and Industrial Technology Development Organization (NEDO). Computational resource of AI Bridging Cloud Infrastructure $(\mathrm{ABCI})$ provided by National Institute of Advanced Industrial Science and Technology (AIST) was used.

\section{REFERENCES}

[1] Takuya Akiba, Shotaro Sano, Toshihiko Yanase, Takeru Ohta, and Masanori Koyama. 2019. Optuna: A next-generation hyperparameter optimization framework. In ACM SIGKDD International Conference on Knowledge Discovery \& Data Mining. ACM, 2623-2631.

[2] Johannes Bader and Eckart Zitzler. 2011. HypE: An algorithm for fast hypervolume-based many-objective optimization. Evolutionary Computation 19, 1 (2011), 45-76

[3] James Bergstra, Rémi Bardenet, Yoshua Bengio, and Balázs Kégl. 2011. Algorithms for Hyper-Parameter Optimization. In Advances in Neural Information Processing Systems. 2546-2554.

[4] James Bergstra, Brent Komer, Chris Eliasmith, Dan Yamins, and David D Cox. 2015. Hyperopt: a python library for model selection and hyperparameter optimization. Computational Science \& Discovery 8, 1 (2015), 014008.

[5] James Bergstra, Daniel Yamins, and David Cox. 2013. Making a Science of Model Search: Hyperparameter Optimization in Hundreds of Dimensions for Vision Architectures. In International Conference on Machine Learning. 115-123.

[6] Dimo Brockhoff, Tobias Friedrich, and Frank Neumann. 2008. Analyzing hypervolume indicator based algorithms. In International Conference on Parallel 
Problem Solving from Nature. Springer, 651-660.

[7] Ran Cheng, Yaochu Jin, Markus Olhofer, and Bernhard Sendhoff. 2016. A reference vector guided evolutionary algorithm for many-objective optimization. IEEE Transactions on Evolutionary Computation 20, 5 (2016), 773-791.

[8] Tinkle Chugh, Yaochu Jin, Kaisa Miettinen, Jussi Hakanen, and Karthik Sindhya. 2018. A Surrogate-assisted Reference Vector Guided Evolutionary Algorithm for Computationally Expensive Many-objective Optimization. IEEE Transactions on Evolutionary Computation 22, 1 (2018), 129-142.

[9] Tinkle Chugh, Karthik Sindhya, Jussi Hakanen, and Kaisa Miettinen. 2019. A survey on handling computationally expensive multiobjective optimization problems with evolutionary algorithms. Soft Computing 23, 9 (2019), 3137-3166.

[10] Tinkle Chugh, Karthik Sindhya, Kaisa Miettinen, Yaochu Jin, Tomas Kratky, and Pekka Makkonen. 2017. Surrogate-assisted evolutionary multiobjective shape optimization of an air intake ventilation system. In IEEE Congress on Evolutionary Computation. 1541-1548.

[11] Tinkle Chugh, Chaoli Sun, Handing Wang, and Yaochu Jin. 2020. SurrogateAssisted Evolutionary Optimization of Large Problems. In High-Performance Simulation-Based Optimization. Springer, 165-187.

[12] Kalyanmoy Deb, Amrit Pratap, Sameer Agarwal, and TAMT Meyarivan. 2002. A fast and elitist multiobjective genetic algorithm: NSGA-II. IEEE Transactions on Evolutionary Computation 6, 2 (2002), 182-197.

[13] Kalyanmoy Deb, Lothar Thiele, Marco Laumanns, and Eckart Zitzler. 2002. Scalable multi-objective optimization test problems. In IEEE Congress on Evolutionary Computation, Vol. 1. IEEE, 825-830.

[14] Jinliang Ding, Cuie Yang, Yaochu Jin, and Tianyou Chai. 2017. Generalized multitasking for evolutionary optimization of expensive problems. IEEE Transactions on Evolutionary Computation 23, 1 (2017), 44-58.

[15] Andreia P Guerreiro, Carlos M Fonseca, and Luís Paquete. 2016. Greedy hypervolume subset selection in low dimensions. Evolutionary Computation 24, 3 (2016), 521-544.

[16] Dan Guo, Yaochu Jin, Jinliang Ding, and Tianyou Chai. 2018. Heterogeneous ensemble-based infill criterion for evolutionary multiobjective optimization of expensive problems. IEEE Transactions on Cybernetics 49, 3 (2018), 1012-1025.

[17] Simon Huband, Luigi Barone, Lyndon While, and Phil Hingston. 2005. A scalable multi-objective test problem toolkit. In International Conference on Evolutionary Multi-Criterion Optimization. Springer, 280-295.

[18] Donald R Jones, Matthias Schonlau, and William J Welch. 1998. Efficient global optimization of expensive black-box functions. Fournal of Global Optimization
13, 4 (1998), 455-492.

[19] Joshua Knowles. 2006. ParEGO: a hybrid algorithm with on-line landscape approximation for expensive multiobjective optimization problems. IEEE Transactions on Evolutionary Computation 10, 1 (2006), 50-66.

[20] Zhichao Lu, Ian Whalen, Vishnu Boddeti, Yashesh Dhebar, Kalyanmoy Deb, Erik Goodman, and Wolfgang Banzhaf. 2019. NSGA-Net: Neural Architecture Search Using Multi-Objective Genetic Algorithm. In The Genetic and Evolutionary Computation Conference. 419-427.

[21] Michael D McKay, Richard J Beckman, and William J Conover. 1979. Comparison of three methods for selecting values of input variables in the analysis of output from a computer code. Technometrics 21, 2 (1979), 239-245.

[22] George L Nemhauser, Laurence A Wolsey, and Marshall L Fisher. 1978. An analysis of approximations for maximizing submodular set functions-I. Mathematical Programming 14, 1 (1978), 265-294.

[23] Linqiang Pan, Cheng He, Ye Tian, Handing Wang, Xingyi Zhang, and Yaochu Jin. 2018. A classification-based surrogate-assisted evolutionary algorithm for expensive many-objective optimization. IEEE Transactions on Evolutionary Computation 23, 1 (2018), 74-88.

[24] Wolfgang Ponweiser, Tobias Wagner, Dirk Biermann, and Markus Vincze. 2008. Multiobjective Optimization on a Limited Budget of Evaluations Using ModelAssisted $\mathcal{S}$-Metric Selection. In International Conference on Parallel Problem Solving from Nature. Springer, 784-794.

[25] Jerome Sacks, William J Welch, Toby J Mitchell, and Henry P Wynn. 1989. Design and Analysis of Computer Experiments. Statist. Sci. 4, 4 (1989), 409-435.

[26] Ye Tian, Ran Cheng, Xingyi Zhang, and Yaochu Jin. 2017. PlatEMO: A MATLAB platform for evolutionary multi-objective optimization [educational forum]. IEEE Computational Intelligence Magazine 12, 4 (2017), 73-87.

[27] Christopher KI Williams and Carl Edward Rasmussen. 2006. Gaussian Processes for Machine Learning. MIT Press Cambridge, MA

[28] Oingfu Zhang and Hui Li. 2007. MOEA/D: A multiobjective evolutionary algorithm based on decomposition. IEEE Transactions on Evolutionary Computation 11, 6 (2007), 712-731.

[29] Meng Zhao and Jinlong Li. 2018. Tuning the hyper-parameters of CMA-ES with tree-structured Parzen estimators. In International Conference on Advanced Computational Intelligence. IEEE, 613-618. 